\title{
A coupled-cluster study of the ground-state energy and properties of an anisotropic quantum spin lattice model exhibiting antiferromagnetism in various phases
}

\author{
R. F. Bishop, J. B. Parkinson, and Yang Xian \\ Department of Mathematics, University of Manchester Institute of Science and Technology, \\ P.O. Box 88, Manchester M60 1QD, UK
}

Received December 5, 1990/Accepted December 6, 1990

Summary. We extend the domain of applicability of the coupled-cluster method (CCM) to include quantum-mechanical spin- $-\frac{1}{2}$ systems on discrete lattices. We study the specific case of anisotropic antiferromagnetic interactions described by the nearest-neighbour XXZ-model Hamiltonian. The isotropic version of this model on a two-dimensional (2-d) square lattice is of great current interest as a possible description of the interactions between the electrons in the singly-occupied $d_{x^{2}-y^{2}}$ orbitals on the copper atoms in the ceramic copper oxide materials displaying high-temperature superconductivity. Although very few exact results are known for the 2-d XXZ-model, its 1-d counterpart has been exactly solved by Bethe-ansatz techniques, and we therefore use it here as a benchmark for our new CCM techniques. Even starting with the classical Néel state as the model reference state, we find that the CCM is capable at relatively low levels of truncation of giving accurate values for the ground-state energies. In this regard, we discuss several new CCM truncation hierarchies which have not previously been applied to either atoms and molecules or continuous extended systems. Furthermore, the method gives a good qualitative description of most of the known or anticipated behaviour of the correlation functions, order parameters, and elementary excitations over an entire (zero-temperature) phase, right up to the transition point, as the anisotropy is varied.

Key words: Coupled cluster method - Quantum spin lattice models - XXZmodel Hamiltonian - Low-dimensional anisotropic quantum antiferromagnets Phase transitions - Calculation of staggered magnetization and correlation functions - Ground states and excited states

\section{Introduction}

The recent discovery by Bednorz and Müller [1] of the first of what has since become a larger class of ceramic materials displaying high-temperature superconductivity, has brought about a huge resurgence of interest in models that describe strongly correlated electronic systems. For example, two such different models have been specifically suggested by Anderson [2] for their potential relevance in this context. 
The first of these is the well-known Hubbard model [3], which is a lattice electron model containing two terms, namely a simple single-electron hopping term between lattice sites, and a second term in which the long-range Coulomb interaction potential is replaced by an on-site delta-function type of interaction which allows the electrons to interact only when on the same site. Anderson [2] has suggested that the non-Fermi liquid behaviour away from half-filling on a two-dimensional (2-d) lattice might be at the heart of high-temperature superconductivity. In particular, he has proposed the concept of resonating-valence bond states in which the many-body system is viewed as a bosonic fluid of singlet or triplet pairs of fermions bound to a lattice $[2,4]$. Other similar studies $[5,6]$ have been based on the alternative hypothesis that the superconducting ground state may be generated instead by the electrons themselves condensing onto the 2-d lattice and carrying spontaneous orbital currents.

A second suggestion of Anderson [2] for a lattice model of relevance to high-temperature superconductivity is that of the $2-\mathrm{d}$ spin- $-\frac{1}{2}$ Heisenberg quantum antiferromagnet, which comprises the usual isotropic (quadratic) spin-spin interactions between nearest-neighboür electrons on a square lattice. The physical hypothesis here is that this model may provide a description of the interactions between the electrons in the singly-occupied $d_{x^{2}-y^{2}}$ orbitals on the copper atoms.

In view of the possible practical importance of such above models as those of Hubbard and Heisenberg, it is therefore particularly timely to attempt to study them at a fundamental microscopic level. Furthermore, the last decade or so has seen the development and use of a number of extremely powerful many-body methods, which are now available to explore from first principles the structure and dynamic behaviour of strongly correlated quantum systems [7]. At the forefront amongst these methods in terms of both universality of applicability and accuracy are the coupled cluster method (CCM) [7-15, and see other articles in this volume] which is already well known in quantum chemistry, and the method of correlated basis functions (CBF) [16-26], which aims systematically to extend and improve upon the variational calculations based on trial correlated wavefunctions of the Bijl-Jastrow type [27].

More recently, the diagrammatic parquet (or planar) theory approach [2831] has also been developed to the point where it holds out much promise. This method focuses on the effective two-body interaction and expresses it in terms of a sum of a large and physically interesting class of Feynman diagrams, namely the parquet diagrams, which comprise a particular self-consistent sum of ring, ladder, and vertex correction diagrams for the two-body Green's function. Nevertheless, this planar method has not yet achieved anything like the number of applications that either the CCM (and see other articles in this volume) or the $\mathrm{CBF}$ approach has.

Much of the earlier work on both the CCM and the CBF method has been concerned with the equilibrium properties of such 'standard' and archetypal quantum fluids as the helium liquids, the electron gas (or jellium), and nuclear matter. Of course, the CCM is also particularly well known to quantum chemists for many extremely accurate calculations of the ground-state energies and other properties of a wide selection of atoms and molecules.

Despite what, particularly for the CCM, is now the very large number of successful applications of these methods to a wide diversity of condensed matter and other systems of chemical and physical interest, only relatively few applications have to date been made to study the dynamics of lattice Hamiltonians. These latter are of importance not only in solid-state physics (e.g., the Hubbard 
and Anderson models of strongly correlated electrons, and the Heinsenberg and other models of ferromagnets and antiferromagnets), but also within the context of lattice gauge models for such lattice gauge field theories as quantum electrodynamics (QED) and quantum chromodynamics (QCD).

Within the last two years the CBF method has already begun to be applied to both the lattice Hubbard model $[32,33]$ and to the $\mathrm{U}(1)_{3}$ lattice gauge model of QED [34] in which the electromagnetic field is defined on the links and plaquettes of a 2-d square lattice. Similarly, the CCM has also recently been applied by Paldus and his coworkers $[35,36]$ to the homologous series of cyclic polyenes [37] $\mathrm{C}_{N} \mathrm{H}_{N}$, with $N=4 n+2$ and $n=1,2, \ldots$, which assures the nondegenerate closed-shell character of their ground states. They have studied these one-dimensional (ring) systems both via the simple model Hubbard Hamiltonian described above, and via its more sophisticated counterpart, the PariserParr-Pople Hamiltonian [38], which gives a much more realistic description of the long-range nature of the interelectronic Coulomb repulsion. For $N \rightarrow \infty$ these systems are also of. interest in the context of solid-state physics, where they model both 1-d metals with a half-filled band and, in the case of bond-length alternation, semiconductors. Finally, Roger and Hetherington [39] have very recently also pioneered the application of the CCM to spin lattice systems. Their main interest was in the solid phase of ${ }^{3} \mathrm{He}$, but they also demonstrated the efficacy of the method for calculating the ground-state energy of the isotropic Heisenberg antiferromagnets in 1-d and 2-d.

Our main purpose in the present paper is to undertake a much more detailed CCM study of these quantum antiferromagnetic systems. In particular, we introduce an anisotropy into the interaction, and study the (zero-temperature) phase transitions with respect to the anisotropy parameter. Furthermore, we study other ground-state properties and correlation functions and excitation energies as well as the ground-state energy itself. One of our main findings is that not only does the CCM give good quantitative descriptions of these quantities over an entire antiferromagnetic phase, but it also gives a qualitatively correct description of the phase change itself as the anisotropy is varied.

Although, as we have indicated above, much of the recent upsurge in interest in lattice models has been in 2-d, in connection with the planar structures deemed to be of relevance to high-temperature superconductivity, it is nevertheless useful, for several reasons, for us to consider 1-d models initially in our attempt to apply CCM techniques to them. In the first place, 1-d models of many different phenomena have a long history of study by theoretical physicists and chemists (and see, e.g., Refs. [40-49]), and hence there are many results with which to compare. Some of the basic motivations behind such theoretical studies are described below.

Secondly, various such 1-d chain models have the great theoretical advantage of exact integrability. They may therefore be used as known benchmarks against which to test the results from such more general microscopic methods as the CCM. Many of these 1-d integrable models have been solved by Bethe-ansatz techniques $[40,50,51]$ and their more modern related algebraic counterparts, namely the quantum inverse scattering (or quantum spectral transform) method of Faddeev and his co-workers [52,53]. Also of interest in this regard is that various seemingly different integrable models can be brought into much closer relationship. For example, the Bethe-ansatz technique was first introduced [50] some sixty years ago to solve the 1-d spin- $-\frac{1}{2}$ Heisenberg model. It was later successfully applied [54-56] to the anisotropic spin- $\frac{1}{2}$ XXZ model in 1-d, which 
forms the focus of interest in the present work. On the other hand, for example, the method has also been applied to the 1-d $N$-site Hubbard model, both in the $N \rightarrow \infty$ limit (of interest, for example, as a model of the quasi-one-dimensional conductor polyacetylene) where exact closed-form analytic solutions are available [57], and for finite $N$ (of interest, for example, as a model of the cyclic polyenes) where only numerical solutions exist [58].

A third reason for emphasising 1-d spin models here is related to the previous discussion. Thus, it is well known [40] that in 1-d not only can fermionic models be transformed into bosonic models, but spin models can also be related to either an equivalent fermionic or bosonic description. Such interrelationships and equivalences between various $1-d$ models have already been used by various authors to predict the behaviour of a variety of nonintegrable (e.g., spin-1) lattice models [59-61]. Thus, we may also expect our present work on spin models to have strong overlaps with comparable models of strongly correlated electronic systems and, indeed, also with nonlinear field-theoretical models.

Fourthly, and finally, although 1-d models were originally studied as simpler proxies for their counterparts in 3-d, a large number of materials are now known to be either quasi-1-d or quasi-2-d with respect to an array of fascinating properties which they exhibit. Many of the earliest of these quasi-1-d extended systems were polymers comprising long chains of conjugated double bonds. Polyacetylene films provide a specific experimental system here, and much theoretical work has been expended on understanding the magnetic, optical and electric properties of pristine and lightly-doped trans-polyacetylene, for example. The cyclic polyene homologous series, to which the CCM has already been applied [35,36], are the finite 1-d analogues in this respect. Their lowest member represents the $\pi$-electronic model of the prototypical aromatic compound, namely the benzene molecule, $\mathrm{C}_{6} \mathrm{H}_{6}$.

Nowadays, there are also many materials which are known to behave to a greater or lesser degree as quasi-1-d spin systems, exhibiting either ferromagnetic or antiferromagnetic properties. For example, neutron scattering experiments [62] on $\mathrm{CPC}$ (dichlorobispyridine copper), $\mathrm{CuCl}_{2} \cdot 2 \mathrm{~N}\left(\mathrm{C}_{5} \mathrm{D}_{5}\right)$, have been interpreted as giving strong evidence that this material provides a rather accurate representation of a spin $-\frac{1}{2} 1-\mathrm{d}$ (isotropic) Heisenberg quantum antiferromagnet. Similarly, $\mathrm{CsCoCl}_{3}$ (and $\mathrm{CsCoBr}$ ) on the one hand and $\mathrm{Cs}_{2} \mathrm{CoCl}_{4}$ on the other are believed to be good physical realizations of $1-d$ spin- $\frac{1}{2}$ quantum antiferromagnets of $\mathrm{XXZ}$ type, showing high anisotropy into the Ising-like and planar (XY-model)-like regimes respectively. In the same vein, $\mathrm{CoCl}_{2} \cdot 2 \mathrm{D}_{2} \mathrm{O}$ is believed to model the comparable $1-\mathrm{d}$ spin- $\frac{1}{2}$ Ising-like quantum ferromagnet. Other materials also model 1-d systems with higher spin values. For example, $\mathrm{CsNiCl}_{3}$ and NENP (nickel(2) ethylenediamine nitrito perchlorate), $\mathrm{Ni}\left(\mathrm{C}_{2} \mathrm{H}_{8} \mathrm{~N}_{2}\right)_{2} \mathrm{NO}_{2}\left(\mathrm{ClO}_{4}\right)$, are both quasi-1-d spin-1 antiferromagnets, and $\mathrm{CsNiF}_{3}$ is a quasi-1-d spin-1 ferromagnet. All three materials are believed to be well represented by the spin-1 Heisenberg model (with additional easy-plane crystal field anisotropy). Finally, TMMC (tetramethylammonium manganese trichloride), $\left(\mathrm{CH}_{3}\right)_{4} \mathrm{NMnCl}_{3}$, is a quasi-1-d spin- $-\frac{5}{2}$ (and hence quasi-classical due to the high spin value) antiferromagnet of nearly Heisenberg type, but with a small anisotropy into the planar (XY-model)-like regime. Many other examples also exist.

Finally, we also point out that while some of these materials are only moderately one-dimensional in nature, others provide extremely accurate representations of $1-\mathrm{d}$ systems. For example, the magnetic $\mathrm{Ni}^{2+}$ ions in the material 
NENP form chains due to the strong super-exchange coupling between the ions. The ratio of interchain to intrachain couplings for this material is confidently estimated to be appreciably less than $0.1 \%$.

The XXZ-model Hamiltonian is first briefly reviewed in Sect. 2, before we describe the application of coupled cluster (CC) techniques to it. We recall that one of the key distinguishing features of the CCM is the underlying similarity transformation that is induced by the cluster correlation (creation) operator $S$ (which is more usually denoted as $T$ in the quantum chemistry literature). We recall further that this aspect implies that the CCM provides basically a biorthogonal (rather than an orthogonal) formulation of the quantum manybody problem. The explicit Hermitian conjugacy of the corresponding bra and ket vectors is thus not manifestly maintained at each level of approximation, for reasons that have been well described elsewhere [14, 15, 63, 64]. Accordingly, we first describe in Sect. 3 the usual CC parametrization of the ground energy eigenket. Although a knowledge of the ket state suffices to evaluate the groundstate energy of the system, other properties require a knowledge of the bra state also. Its corresponding parametrization in what we nowadays refer to as the normal CCM (NCCM) - to distinguish it from the extended (ECCM) version of the CCM first introduced by Arponen [14, 15, 63, 64] -is then described in Sect. 4.

In Sects. 3 and 4 we describe both how standard CCM approximation schemes, which are already well-known in quantum chemistry, can be tailored to the spin lattice problems at hand, and, furthermore, how several rather intuitive and appealing new schemes may also be introduced. The usefulness of each of these schemes is demonstrated in Sect. 3 by comparing their predictions for the ground-state energy with known exact results. In particular, we show how a definite signal of the known antiferromagnetic phase change in $1-\mathrm{d}$ is observed in specific CCM approximations.

We then turn in Sect. 5 to the calculation of the staggered magnetization and of the correlation function and its corresponding associated order parameter. These quantities provide much more demanding tests for an approximate wavefunction than the ground-state energy. In particular, they provide a measure of the presence or absence of long-range antiferromagnetic order in the system. We observe once more that their behaviour as functions of the anisotropy in the model Hamiltonian is also strongly suggestive of a phase transition in the same CCM approximations and at the same corresponding values of the anisotropy parameter as was observed in the ground-state energy calculations of Sect. 3.

As a final piece of evidence that the previously observed behaviour does indeed represent a phase transition, and not simply a mathematical breakdown of the particular CCM approximation schemes adopted, we discuss the corresponding NCCM parametrization of the excited states in Sect. 6. We observe now that whereas the (approximate) excitation spectrum has a gap at the boundaries of the Brillouin zone for values of the anisotropy parameter where there is nonzero long-range order as measured by the earlier calculations of the staggered magnetization and the order parameter, this gap disappears precisely at the same critical value where the order disappears, and which signals the phase transition.

Our results on these 1-d spin- $\frac{1}{2}$ chains are summarized and discussed in Sect. 7. In view of their considerable success in describing both the quantitative behaviour of the ground-state energy and the correct qualitative behaviour of the long-range correlations and the elementary excitations over an entire phase right 
up to the transition point, we also consider their generalizations to lattices of higher dimensionality and to spins, $s>\frac{1}{2}$. In both of these cases almost no exact results are known. Furthermore, both extensions are of considerable topical interest, in the former case with regard to the suggested relevance of the 2-d spin- $-\frac{1}{2}$ model to high-temperature superconductivity [2], and in the latter case with regard to the remarkable conjecture of Haldane [59] concerning the qualitatively different nature of the zero-temperature phase diagram for the spin- 1 case in comparison to its spin- $-\frac{1}{2}$ counterpart.

\section{The XXZ model: some preliminaries}

The most general XXZ-model Hamiltonian comprises $N$ quantum-mechanical spins $\vec{s}_{i} \equiv\left\{s_{i}^{\alpha} ; \alpha=x, y, z\right\}$ on the sites $\{i\}$ of a given regular lattice in $d$ dimensions. Periodic boundary conditions are usually assumed for the lattice, and in any case we are generally interested in the infinite lattice limit, $N \rightarrow \infty$. The spins interact via the anisotropic nearest-neighbour Hamiltonian:

$$
H=\frac{1}{2} \sum_{i=1}^{N} \sum_{\varrho=1}^{\nu}\left(s_{i}^{x} s_{i+\varrho}^{x}+s_{i}^{y} s_{i+\varrho}^{y}+\Delta s_{i}^{z} s_{i+\varrho}^{z}\right),
$$

where the sum on $\varrho$ runs over all $v$ nearest neighbours. For example, the coordination numbers $v$ for the $1-\mathrm{d}$ chain and the 2-d square lattice are $v=2,4$, respectively. The Hamiltonian of Eq. (1) contains as three special cases the Ising model $(\Delta \rightarrow \infty)$, the (isotropic) Heisenberg model $(\Delta=1)$, and the planar (or $\mathrm{XY}-)$ model $(\Delta=0)$.

The spins obey the usual $\mathrm{SU}(2)$ angular momentum algebra:

$$
\left[s_{j}^{\alpha}, s_{k}^{\beta}\right]=i \delta_{j k} s_{k}^{\gamma},
$$

where $(\alpha, \beta, \gamma)$ is any cyclic permutation of the indices $(x, y, z)$. From the commutation relation of Eq. (2) it is easy to show that:

$$
\left[H, s_{T}^{z}\right]=0 \text {, }
$$

where $\vec{s}_{T}$ is the total spin operator:

$$
\vec{s}_{T}=\sum_{i=1}^{N} \vec{s}_{i}
$$

The energy eigenstates may thus be chosen to be simultaneous eigenstates of the $z$-component of total spin, $s_{T}^{z}$, which is hence a good quantum number. In general, the spin quantum number $s$ may take any of the usual integral or half-integral values:

$$
\vec{s}_{j}^{2}=s(s+1) ; \quad s=\frac{1}{2}, 1, \frac{3}{2}, \ldots
$$

For present purposes we shall mostly be interested in the case $s=\frac{1}{2}$. We shall also concentrate almost wholly on the 1-d chain.

It is clear that the ground state of the classical counterpart of Eq. (1) is ferromagnetic, with all spins aligned along the $z$-axis, for all lattices when $\Delta \leqslant-1$. The corresponding classical ground-state energy is $E_{c l}=\frac{1}{2} N v \Delta s^{2}$. Conversely, in the case $\Delta>-1$, the classical ground state is antiferromagnetic for all bipartite lattices. In this latter case all of the spins are again aligned along a specific axis, but in opposite directions on the two sublattices. Thus, for $|\Delta|<1$ the classical antiferromagnetic magnetization axis is along some arbitrary direc- 
tion in the $x y$-plane, whereas for $\Delta>1$ it is along the $z$-axis. The corresponding classical ground-state energies are $E_{c l}=-\frac{1}{2} N v s^{2}$ for $|\Delta|<1$, and $E_{c l}=-\frac{1}{2} N v \Delta s^{2}$ for $\Delta \geqslant 1$. We note that this classical Néel state in which all nearest-neighbour pairs have opposed spins is only possible for bipartite lattices, such as the 1-d chain and the 2-d square lattice. By contrast, the 2-d triangular lattice is not bipartite, and provides an example of where such a simple antiferromagnetic means of minimizing the energy of Eq. (1) in terms of two sublattices is frustrated.

We note that whereas the perfectly aligned ferromagnetic state with all spins pointing in the same direction along the $z$-axis is an exact eigenstate of the quantum-mechanical Hamiltonian of Eq. (1) as well as of its classical counterpart, the same is not true of the Néel state. In the Néel state with $z$-axis as magnetization axis, we have $s_{T}^{z}=0$. The $x y$ part of the Hamiltonian now induces quantum fluctuations in the Néel state which must be included to obtain the exact ground state. Nevertheless, it is clear that the $x y$-planar term in $H$ can only act to exchange spins on different sublattices, and hence also that $s_{T}^{z}$ is conserved as we have already seen. We shall therefore work wholly within the $s_{T}^{z}=0$ subspace, since this is known to include the exact antiferromagnetic ground state, at least for the $s=\frac{1}{2}$ XXZ-model with $\Delta>-1$ in 1-d. In fact, a stronger theorem has been proved [65] for the isotropic $(\Delta=1)$ Heisenberg case in any number of dimensions, namely that the ground state of a discrete spin $-\frac{1}{2}$ system is nondegenerate (and hence an $s_{T}=0$ singlet) for any bipartite lattice with equal numbers $N / 2$ of spins on each equivalent sublattice (i.e., such that the Heisenberg interactions are ferromagnetic between spins on the same sublattice and antiferromagnetic between those on different sublattices).

The starting point for any CCM parametrization is an uncorrelated model state $|\Phi\rangle$, and for the present spin $-\frac{1}{2}$ XXZ-model on a bipartite lattice we shall henceforth always choose $|\Phi\rangle$ to be the equally populated two-sublattice Néel state with $z$-axis chosen as quantization axis, and hence with $s_{T}^{z}=0$. It is particularly convenient [39] to perform a notional rotation of $180^{\circ}$ about the $y$-axis on one sublattice, say the up-sublattice, so that in this so-called Néel basis the model state has all of the spins in the "down" direction. Thus, on the up-sublattice we have the transformation $s_{x} \rightarrow-s_{x}, s_{y} \rightarrow s_{y}, s_{z} \rightarrow-s_{z}$.

For the $s=\frac{1}{2}$ system to which we now restrict ourselves we also introduce the usual Pauli spin matrices, $\sigma_{k}^{\alpha}=2 s_{k}^{\alpha} ; \alpha=x, y, z$. We also introduce the usual raising and lowering operators (albeit with a nonstandard normalization constant), such that in the Néel basis $\sigma_{k}^{ \pm} \equiv \frac{1}{2}\left(\sigma_{k}^{x} \pm i \sigma_{k}^{y}\right)$ on the down-sublattice, and $\sigma_{k}^{ \pm} \equiv \frac{1}{2}\left(-\sigma_{k}^{x} \pm i \sigma_{k}^{\nu}\right)$ on the rotated up-sublattice. We note that the Néel rotation leaves the algebra of Eq. (2) unchanged, so that on all sites we have the equivalent commutation relations:

$$
\left[\sigma_{j}^{+}, \sigma_{k}^{-}\right]=\sigma_{j}^{z} \delta_{j k} ; \quad\left[\sigma_{j}^{z}, \sigma_{\bar{k}}^{+}\right]= \pm 2 \sigma_{j}^{ \pm} \delta_{j k} .
$$

The Hamiltonian of Eq. (1) may now be rewritten in the Néel basis as:

$$
H=-\sum_{i=1}^{N} \sum_{e=1}^{v}\left[\frac{1}{4}\left(\sigma_{i}^{+} \sigma_{i+e}^{+}+\sigma_{i}^{-} \sigma_{i+e}^{-}\right)+(\Delta / 8) \sigma_{i}^{z} \sigma_{i+e}^{z}\right] \text {. }
$$

We note that in the Néel basis we now have that $\sigma_{k}^{-}|\Phi\rangle=0$ for all $k$, while $\sigma_{k}^{+}|\Phi\rangle$ is a state with the $k$ th spin reversed with respect to the model Néel state. Thus, the Néel state now acts as a cyclic vector for the sets of operators $\left\{\sigma_{k}^{+}\right\}$ and $\left\{\sigma_{k}\right\}$, which play the respective roles of complete sets of single-spin creation 
and destruction operators in relation to it. More specifically, we have that if we denote the wavefunctions for a single spin to be in the up and down states (in the Néel basis) as $|+\rangle$ and $|-\rangle$ respectively, then:

$$
\sigma^{z}| \pm\rangle= \pm| \pm\rangle ; \quad \sigma^{ \pm}|\mp\rangle=| \pm\rangle ; \quad \sigma^{ \pm}| \pm\rangle=0 .
$$

We conclude these introductory remarks with a brief discussion of some of the exact results that are known for the XXZ-model. For the $s=\frac{1}{2} 1-\mathrm{d}$ Heisenberg ( $\Delta=1$ ) chain, the exact eigenstates were first obtained for arbitrary $N$ as long ago as 1931 by Bethe [50]. Nevertheless, these exact wavefunctions were (and remain) extremely difficult to manipulate to obtain quantities of physical interest. For example even the ground-state energy of the infinite chain was not obtained until about seven years later, when Hulthén [66] made use of an integral equation approach. Even more importantly, the uncertainty surrounding the nature of the long-range order contained in these wavefunctions lasted much longer. In particular, when coupled with the inapplicability of the Bethe-ansatz approach to lattices of higher dimensionality, this continuing doubt led both Anderson [67] and Kubo [68] independently to the approximate description of these systems in terms of the so-called spin-wave theory. Finally at the ground-state level, the Bethe-ansatz method was extended by Orbach [54] in 1958 to deal with the $s=\frac{1}{2}$ XXZ-model in 1-d considered here.

The low-lying excitations of the $s=\frac{1}{2}$ Heisenberg chain were first investigated by des Cloiseaux and Pearson [69] in 1962. They used a generalization of the earlier approach of Hulthén [66]. A detailed description of what became known as the antiferromagnetic spin-wave excitations of the anisotropic XXZ-model $s=\frac{1}{2}$ chain was given a few years later by des Cloiseaux and Gaudin [70]. On the other hand, it was not until as late as 1981 that Faddeev and Takhtajan [71] resolved the confusion and misunderstanding that had heretofore existed in connection with the precise nature of the spin-wave excitations given by the Bethe-ansatz techniques. They established for the $s=\frac{1}{2}$ Heisenberg antiferromagnet that the actual spin of an elementary spin wave on the 1-d chain is itself equal to one-half, by contrast with the singlet $(s=0)$ or triplet $(s=1)$ values that previous authors had described. In particular, by making contact between the Bethe-ansatz techniques and the then recently invented quantum inverse scattering method $[52,53]$, Faddeev and his coworkers showed how this elementary spin- $\frac{1}{2}$ spin-wave excitation could be interpreted as a single kink-soliton. Furthermore, they demonstrated how all actual physical states have integral values of the spin, and hence comprise an even number of spin waves or kinks (and antikinks).

We summarize below the main exact results that have emerged out of the work discussed above for the $s=\frac{1}{2} \mathrm{XXZ}$-model in 1-d. In the ferromagnetic $\left(s_{T}^{z}= \pm \frac{1}{2} N\right)$ regime, $\Delta \leqslant-1$, the exact ground-state energy is given by:

$$
\frac{E_{g}}{N}=\frac{1}{4} \Delta ; \quad \Delta \leqslant-1,
$$

just as in the classical case. Precisely at the point $\Delta=-1$ there is a first-order phase transition to a 'critical' antiferromagnetic phase. This phase persists over the entire range $|\Delta| \leqslant 1$ of the anisotropy parameter. Since it includes the $\Delta=0$ case we refer to it as the planar-like (or XY-model-like) phase. Its ground-state energy is given by the exact expression:

$$
\begin{array}{r}
\frac{E_{g}}{N}=\frac{1}{4} \cos \theta-\frac{1}{2} \sin ^{2} \theta \int_{-\infty}^{\infty} \frac{d \omega}{\cosh (\pi \omega)[\cosh (2 \theta \omega)-\cos \theta]} ; \quad|\Delta| \leqslant 1, \\
0 \leqslant \theta \equiv \cos ^{-1} \Delta \leqslant \pi .
\end{array}
$$


Equation (10) can be specifically evaluated for the planar model, to give:

$$
\frac{E_{g}}{N}=-\frac{1}{\pi} ; \quad \Delta=0
$$

Whereas the critical phase which exists for $|\Delta|<1$ has a $\left(s_{T}^{z}=0\right)$ nondegenerate ground state, at $\Delta=1$ the system undergoes another phase change to a new Ising-like antiferromagnetic phase which persists over the entire range $\Delta>1$. This phase has a doubly-degenerate ground state, but still with $s_{T}^{z}=0$. The ground-state energy changes continuously and smoothly at $\Delta=1$, and is given for $\Delta \geqslant 1$ by the exact expression:

$$
\frac{E_{g}}{N}=\frac{1}{4} \cosh \gamma-\frac{1}{2} \sinh \gamma\left[1+4 \sum_{m=1}^{\infty}\left(1+e^{2 m \gamma}\right)^{-1}\right] ; \quad 1 \leqslant \Delta \equiv \cosh \gamma .
$$

Direct evaluation from Eq. (12) shows that in the Heisenberg and Ising limits the ground-state energy is given respectively as:

$$
\begin{aligned}
& \frac{E_{g}}{N}=\frac{1}{4}-\ln 2 ; \quad \Delta=1, \\
& \frac{E_{g}}{N} \underset{\Delta \rightarrow \infty}{\longrightarrow}-\frac{1}{4}\left(\Delta+\frac{1}{\Delta}\right) .
\end{aligned}
$$

What principally distinguishes the two antiferromagnetic phases of the $s=\frac{1}{2}$ XXZ-model chain in 1-d are their long-range order properties. These may conveniently be summarized in terms of the spin-spin correlation function $G_{n}$ defined by the ground-state expectation value:

$$
G_{n} \equiv\left\langle\overrightarrow{\boldsymbol{\sigma}}_{i} \cdot \overrightarrow{\boldsymbol{\sigma}}_{i+n}\right\rangle,
$$

between spins $n$ sites apart on the chain. We note that the translational variance implied by the imposition of periodic boundary conditions ensures that $G_{n}$ is independent of absolute position $i$ on the chain. We may define an associated order parameter $\mu$ as:

$$
\mu=\lim _{n \rightarrow \infty}\left|G_{n}\right| .
$$

We may also define the staggered magnetization $\vec{M}$ as:

$$
\vec{M} \equiv\left\langle\overrightarrow{\boldsymbol{\sigma}}_{i}\right\rangle .
$$

Since both $G_{n}$ and $\vec{M}$ are defined in terms of operators $\overrightarrow{\boldsymbol{\sigma}}_{i}$ given in the Néel basis, they do not have the alternating signs associated with the sublattice structure in the antiferromagnetic regime.

The Ising-like $(\Delta>1)$ phase is now characterized by the presence of longrange order, such that $\mu \neq 0$ and $M \neq 0$. Furthermore, the spin-spin correlation function $G_{n}$ decays exponentially to its long-range limit. By contrast, both the order parameter $\mu$ and the staggered magnetization $M$ are zero in the planar-like $(|\Delta|<1)$ phase. Nevertheless, this latter phase is 'critical' in the usual sense that the correlations decay algebraically (i.e., with power-law behaviour) to zero, rather than exponentially. Thus, in the two antiferromagnetic phases we have the exact behaviour:

$$
G_{n} \underset{n \rightarrow \infty}{\longrightarrow} \begin{cases}\mu+A \ell^{-n} ; & \Delta>1 \\ B n^{-\beta} ; & |\Delta|<1,\end{cases}
$$


where the constants $\mu, A, B$ and $\ell$, as well as the exponent $\beta>0$, all depend on the anisotropy parameter $\Delta$. The order parameter itself has the limiting behaviour:

$$
\mu \rightarrow \begin{cases}1 ; & \Delta \rightarrow \infty \\ 0 ; & \Delta \rightarrow 1\end{cases}
$$

The other main qualitative difference between the two antiferromagnetic phases concerns the presence or absence of a gap in the excitation spectrum. Thus the excited states are generally described in terms of a dispersion law $\varepsilon(k)$ for the excitation energy as a function of the momentum variable $k$ within the first Brillouin zone, namely $-\pi<q \leqslant \pi$ in 1-d. Faddeev and Takhtajan [71] have nevertheless indicated that the fundamental excitation is that of a single $s_{T}=\frac{1}{2}$ kink with momentum $0 \leqslant q \leqslant \pi$. The exact excitation energy of this single-kink state in the critical (planar-like) phase is given as:

$$
\varepsilon(q)=\frac{\pi}{2}\left(\frac{\sin \theta}{\theta}\right) \sin (q) ; \quad 0 \leqslant \theta \equiv \cos ^{-1} \Delta \leqslant \pi, \quad 0 \leqslant q \leqslant \pi .
$$

The spectrum of Eq. (18) is such that $\varepsilon(\pi)=0$, and is hence referred to as gapless. By contrast, in the Ising-like phase the single-kink state has excitation energy:

$$
\varepsilon(q)=\frac{K_{1}}{\pi} \sinh \gamma\left(1-k_{1}^{2} \cos ^{2} q\right)^{\frac{1}{2}} ; \quad 1<\Delta \equiv \cosh \gamma,
$$

where the parameter $k_{1}$ is given in terms of the complete elliptic integrals $K_{1}$ and $K_{1}^{\prime}$ :

$$
K_{1} \equiv \int_{0}^{\frac{1}{2} \pi} d \theta\left(1-k_{1}^{2} \sin ^{2} \theta\right)^{-\frac{1}{2}} ; \quad K_{1}^{\prime} \equiv \int_{0}^{\frac{1}{2} \pi} d \theta\left[1-\left(1-k_{1}^{2}\right) \sin ^{2} \theta\right]^{-\frac{1}{2}}
$$

by the relation:

$$
\frac{K_{1}^{\prime}}{K_{1}}=\frac{\gamma}{\pi}
$$

We note that in this Ising-like phase, the excitation spectrum of Eq. (19) displays a nonzero gap at the zone boundary, $\varepsilon(\pi) \neq 0$.

We note finally that the observed physical excitations with $s_{T}^{z}= \pm 1$ are actually formed from two such kink solutions. The excitation energy obeys the exact additive relation:

$$
E_{k}(q)=\varepsilon(k)+\varepsilon(q-k) .
$$

The excitation spectrum is thus actually a continuum, with respect to the momentum parameter $k$, for a fixed value of total momentum, $q$. The previously mentioned antiferromagnetic spin waves $[69,70]$ are more precisely now just described by the lower boundary to this continuum.

\section{CCM parametrization of the ground ket states; ground-state energy}

The exact ground-state ket wavefunction $|\Psi\rangle$ is expressed in the conventional CCM form:

$$
|\Psi\rangle=e^{S}|\Phi\rangle
$$


in terms of the Néel state $|\Phi\rangle$ as our chosen uncorrelated model state. Since we know that $s_{T}^{z}$ is a conserved quantum number, and since we work within the invariant subspace $s_{T}^{z}=0$, the only $n$-body partitions, $S_{n}$, of the correlation operator $S$ that contribute are those which flip an even number of spins with respect to $|\Phi\rangle$ :

$$
S=\sum_{\ell=1}^{L} S_{2 \ell}
$$

where $N=2 L$ is the total (even) number of spins. For the $s=\frac{1}{2}$ case in $1-\mathrm{d}$ which we consider here, we may write:

$$
S_{n+1}=\sum_{i=1}^{N} \sum_{\left\{r_{1}, r_{2}, \ldots, r_{n}\right\}} s_{r_{1} r_{2} \ldots r_{n}}^{(n+1)} \sigma_{i}^{+} \sigma_{i+r_{1}}^{+} \sigma_{i+r_{2}}^{+} \cdots \sigma_{i+r_{n}}^{+} .
$$

The $n$-fold sum in Eq. (25) over the ordered set of indices $\left\{r_{1}, r_{2}, \ldots, r_{n}\right\}$ is over all values of the individual indices between 1 and $N$ compatible with the triple requirements that $1 \leqslant r_{1}<r_{2}<\cdots<r_{n}<N$; that in Eqs. (24) and (25) the only configurations with nonzero weight in the $s_{T}^{z}=0$ sector contain equal numbers of spin-reversals with respect to $|\Phi\rangle$ on even and on odd sites; and the fact that the periodic boundary condition imposes extra symmetries on the configurations (e.g., $s_{n}^{(2)}=s_{N-n}^{(2)}$ ) and that such otherwise equal configurations should only be counted once. We also note that the translational invariance implied by the periodic boundary condition makes the coefficients in Eq. (25) independent of the absolute site index $i$. Henceforth, we work directly in the infinite chain limit, $N=2 L \rightarrow \infty$.

As usual, it is now necessary to consider various approximation schemes for the correlation operator $S$. The standard SUB $n$ truncation scheme is certainly the best known such method. In the present case it simply amounts to replacing the upper limit $L=\frac{1}{2} N$ in Eq. (24) by some (much reduced) value $\ell^{\prime}=\frac{1}{2} n$, where $n$ is even, and hence setting to zero all higher partitions $S_{m}$ of the correlation operator, for $m>n$. The lowest such approximation now is the SUB2 truncation, $S \rightarrow S_{2}$, which is well known in quantum chemistry as the CCD approximation (or, in this case, also the CCSD approximation since $S_{1} \equiv 0$ in the present $s_{T}^{z}=0$ sector), where the initials $\mathrm{S}$ and $\mathrm{D}$ refer to the single- and double-excitation partitions $S_{1}$ and $S_{2}$, respectively. For our present spin- $\frac{1}{2}$ infinite 1 -d chain, we have:

$$
S_{\mathrm{SUB} 2} \equiv S_{2}=\sum_{i=1}^{\infty} \sum_{m=1}^{\infty} b_{2 m-1} \sigma_{i}^{+} \sigma_{i+2 m-1}^{+} .
$$

Clearly, a full SUB2 calculation now involves the determination of the set of parameters $\left\{b_{r} ; r=1,3,5, \ldots\right\}$. We may also define a further SUB2- $n$ subapproximation, with $n$ as an even positive integer, in which we keep only the lowest $\frac{1}{2} n$ double-spin-flip parameters $\left\{b_{r} ; r=1,3,5, \ldots, n-1\right\}$ by truncating the sum over the index $m$ in Eq. (26) at the value $\frac{1}{2} n$.

Other physically motivated schemes are also possible, and it is of interest to examine them simultaneously. For example, the analysis of Faddeev and his co-workers $[52,53,71]$ discussed previously, has clearly indicated the concept of a single-kink excitation as playing a central and fundamental role, from which other excited states may be constructed as multi-kink-antikink-pair solutions. These considerations lead us to investigate the possibility of constructing an approximation scheme in which the basic concept used to decide which configu- 
rations to keep in Eqs. (24) and (25) is not the total number of "wrong" spins (with respect to $|\Phi\rangle$ ) as in the $\mathrm{SUB} n$ scheme, but rather the number of kinks or antikinks.

In this way we are led to what we henceforth denote as the PSUB $n$ scheme, in which at a given $n$ th-order level we retain only those "wrong-spin" configurations which comprise no nore than $n$ "plaquettes". By definition, each plaquette (or "domain") on the 1-d chain is a contiguous cluster of wrong spins which is terminated at each end by a kink or antikink (or "domain wall"), which delimits it from any other plaquette. A single plaquette may be of arbitrary length. By the above definition each plaquette is separated from any other by at least one intervening "correct" spin (i.e., one which is unaltered from its Néel-state value). Each plaquette thus models a kink/antikink pair, and the PSUB $n$ scheme thereby focuses on the number $2 n$ of boundaries between "correct" and "wrong" blocks of spins, rather than on the number of wrong spins themselves.

Thus, in the lowest PSUB1 approximation, we have that the cluster correlation operator $S$ is approximated in the $s_{T}^{z}=0$ sector as:

$$
S \rightarrow S_{\mathrm{PSUB} 1}=\sum_{i=1}^{\infty} \sum_{m=1}^{\infty} g_{2 m} \prod_{\ell=1}^{2 m} \sigma_{i+\ell-1}^{+} .
$$

We may again define a further truncated PSUB1- $n$ subapproximation, in which we keep only single-plaquette configurations of up to $n$ adjacent spin-flips, where $n$ is even. It is thus fully determined by the set of $\frac{1}{2} n$ coefficients $\left\{g_{p} ; p=2,4,6, \ldots, n\right\}$.

A third sequence of approximations, which we denote as the LSUB- $n$ scheme, is based on the localized nature of the interactions themselves and of the excitations that they cause. At the LSUB- $n$ level of approximation, we now retain only those configurations for the correlation operator $S$ which comprise an arbitrary number of spin-flips with respect to the Néel state over a "locale" of size $n$, and which are compatible with $s_{T}^{z}=0$. In the present 1-d chain context, an $n$ th-order locale is simply a string of $n$ immediately adjacent sites. Obviously the LSUB-2 approximation is identical to both of the previous SUB2-2 and PSUB1-2 schemes, whereas in the LSUB-4 and LSUB-6 approximations respectively we replace $S \rightarrow S_{\mathrm{LSUB}-4}$ and $S \rightarrow S_{\mathrm{LSUB}-6}$, where:

$$
\begin{aligned}
S_{\mathrm{LSUB}-4}= & \sum_{i=1}^{\infty}\left(b_{1} \sigma_{i}^{+} \sigma_{i+1}^{+}+b_{3} \sigma_{i}^{+} \sigma_{i+3}^{+}+g_{4} \sigma_{i}^{+} \sigma_{i+1}^{+} \sigma_{i+2}^{+} \sigma_{i+3}^{+}\right), \\
S_{\mathrm{LSUB}-6}= & S_{\mathrm{LSUB}-4}+\sum_{i=1}^{\infty}\left(b_{5} \sigma_{i}^{+} \sigma_{i+5}^{+}+f_{12} \sigma_{i}^{+} \sigma_{i+3}^{+} \sigma_{i+4}^{+} \sigma_{i+5}^{+}\right. \\
& +f_{14} \sigma_{i}^{+} \sigma_{i+2}^{+} \sigma_{i+3}^{+} \sigma_{i+5}^{+}+f_{23} \sigma_{i}^{+} \sigma_{i+1}^{+} \sigma_{i+4}^{+} \sigma_{i+5}^{+} \\
& +f_{25} \sigma_{i}^{+} \sigma_{i+1}^{+} \sigma_{i+3}^{+} \sigma_{i+4}^{+}+f_{34} \sigma_{i}^{+} \sigma_{i+1}^{+} \sigma_{i+2}^{+} \sigma_{i+5}^{+} \\
& \left.+g_{6} \sigma_{i}^{+} \sigma_{i+1}^{+} \sigma_{i+2}^{+} \sigma_{i+3}^{+} \sigma_{i+4}^{+} \sigma_{i+5}^{+}\right) .
\end{aligned}
$$

Undoubtedly, other truncation hierarchies may also commend themselves, but in the rest of this paper we discuss only the results obtained by the above three schemes. In each case the procedure to evaluate the retained cluster configuration coefficients is the same. Thus, the ground-state Schrödinger equation, $H|\Psi\rangle=E_{g}|\Psi\rangle$, is first written in the usual CCM similarity-transformed form:

$$
e^{-S} H e^{S}|\Phi\rangle=E_{g}|\Phi\rangle
$$


Taking the inner product of Eq. (30) with the Néel state $|\Phi\rangle$ itself rather easily leads to the exact result for the ground-state energy of the 1-d chain:

$$
E_{g} / N=-\frac{1}{4}\left(\Delta+2 b_{1}\right)
$$

where $b_{1} \equiv s_{1}^{(2)}$, as above. Similarly, a set of coupled nonlinear algebraic equations for the various cluster configuration coefficients is obtained by taking the inner product of Eq. (30) in turn with the respective wavefunctions corresponding to each configuration retained in Eqs. (24) and (25) by the specific approximation. After deriving the resulting exact such equations the coefficients of any configurations outside the retained set are then set to zero in the usual way, to have a closed set of equations.

The actual evaluation of the coupled set of equations for each approximation scheme is now straightforward in principle although often cumbersome in practice. We quote only the final results. Thus, in the first place, the full SUB2 equations may be evaluated as follows:

$$
\begin{gathered}
2 \Delta b_{1}-1+5 b_{1}^{2}-2 \sum_{n=1}^{\infty} b_{2 n-1}\left(b_{2 n-1}+b_{2 n+1}\right)=0, \\
2\left(\Delta+2 b_{1}\right) b_{2 m-1}-\frac{1}{2} \sum_{n=1}^{\infty} b_{2 n-1}\left(b_{|2 m-2 n-1|}+b_{|2 m-2 n+1|}\right. \\
\left.\quad+b_{2 m+2 n-1}+b_{2 m+2 n-3}\right)=0 ; \quad m \geqslant 2 .
\end{gathered}
$$

The lowest subapproximation, namely SUB2-2, immediately gives from Eq. (32a) the equation $3 b_{1}^{2}+2 \Delta b_{1}-1=0$, and hence from Eq. (31) the corresponding estimate for the ground-state energy:

$$
\frac{E_{g}}{N}=-\frac{1}{12}\left[\Delta+2\left(\Delta^{2}+3\right)^{\frac{1}{2}}\right] ; \quad \text { SUB2-2. }
$$

It is interesting to note that even this extremely simple SUB2-2 subapproximation has the correct asymptotic form of Eq. (13b) in the Ising-model limit. Curiously, it is also exact at the $\Delta=-1$ transition point to the ferromagnetic state, where $E_{g} / N=-\frac{1}{4}$, from Eq. (9). For the particular Heisenberg $(\Delta=1)$ and XY-planar $(\Delta=0)$ cases, Eq. (33) yields the respective SUB2-2 results, $E_{g} / N=-\frac{5}{12} \approx-0.417$ and $-\sqrt{3} / 6 \approx-0.289$. These values may be compared with their exact counterparts from Eqs. (11) and (13a), namely -0.443 and -0.318 respectively to the same three significant figure level of accuracy.

Numerical investigation of the higher-order SUB2- $n$ subapproximations indicates that, at least for values $\Delta \geqslant 0$, the resulting sequence of approximants for $E_{g} / N$ converges very rapidly as a function of $n$. More interestingly, the full SUB2 Eqs. (32a,b) may also be solved exactly by Fourier transform techniques, to yield the explicit solution:

$$
b_{2 m-1}=\frac{K}{2 \pi} \int_{-\pi}^{\pi} d x\left\{1-f\left(x ; k^{2}\right)\right\} \frac{\cos \left[\left(m-\frac{1}{2}\right) x\right]}{\cos \left(\frac{1}{2} x\right)} ; \quad m \geqslant 1,
$$

where the function $f\left(x ; k^{2}\right)$ is defined as:

$$
f\left(x ; k^{2}\right) \equiv\left\{1-k^{2} \cos ^{2}\left(\frac{1}{2} x\right)\right\}^{\frac{1}{2}}
$$

and where the constants $K$ and $k^{2}$ are defined as:

$$
K \equiv \Delta+2 b_{1} ; \quad k^{2} \equiv\left(1+2 \Delta b_{1}+2 b_{1}^{2}\right) / K^{2} .
$$


A self-consistent equation for the coefficient $b_{1}$ is obtained by putting $m=1$ in Eq. (34). This equation is easily solved numerically. In this way we obtain, for example, the SUB2 estimate $E_{g} / N \approx-0.41862$ for the infinite Heisenberg $(\Delta=1)$ chain. Once again, the full SUB2 approximation reproduces the correct asymptotic form of Eq. (13b) for large values of $\Delta$.

The most interesting feature of the full SUB2 results, however, arises from the fact that real solutions for the coefficients $\left\{b_{r}\right\}$ clearly only exist if $|k| \leqslant 1$. In turn, it is not difficult to show that this condition is fulfilled only for $\Delta \geqslant \Delta_{c}$, where $\Delta_{c} \approx 0.37275$. We show in Fig. 1 the estimates for $E_{g} / N$ versus $\Delta$ for both the SUB2-2 and SUB2 approximation schemes, together with the corresponding exact results from Eqs. (9), (10) and (12). We note that the exact phase transition at $\Delta=1$ is not at all evident from this plot. The absence of a SUB2 solution in the regime below the terminating point, $\Delta<\Delta_{c}$, clearly signals a possible phase transition in the physical system, even though the value of $\Delta_{c}$ is not very close to the exact value of $\Delta=1$. Nevertheless, without further supporting evidence we are certainly not able to rule out the possibility that the terminating point at $\Delta=\Delta_{c}$ in the SUB2 approximation is simply a mathematical breakdown due to an inadequate description of the physical system.

In order to investigate the above point further, we also calculate in Sect. 5 such other properties of the system as the staggered magnetization and the spin-spin correlation function. However, even at this stage where we have only a knowledge of the ground ket state, the long-range behaviour of the double-spinflip configuration coefficients $\left\{b_{r}\right\}$ is rather suggestive that the terminating point might indeed approximate the actual transition which we know occurs at $\Delta=1$.

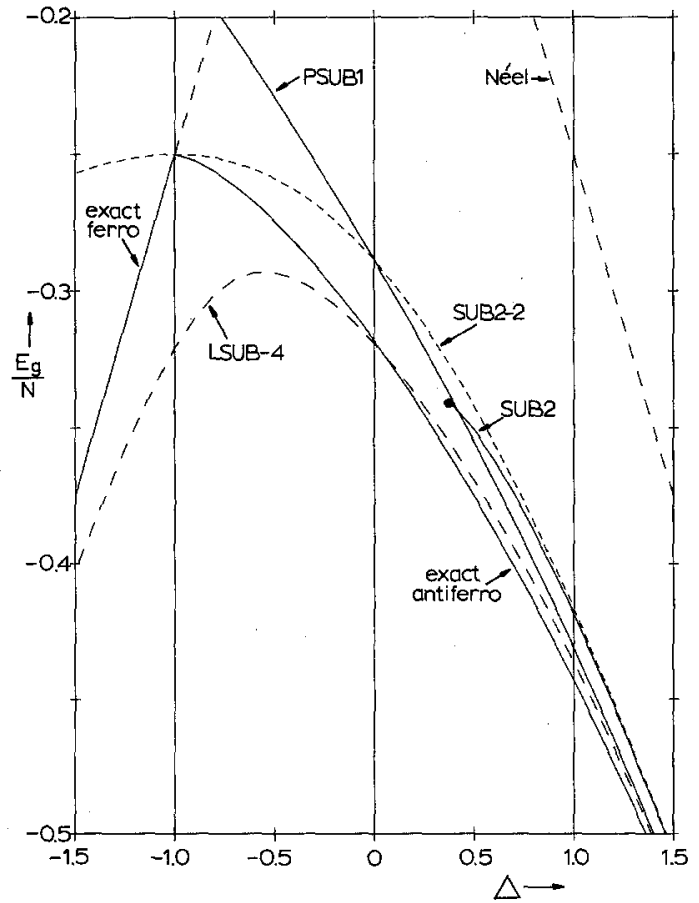

Fig. 1. The ground-state energy per spin, $E_{g} / N$, of the infinite spin- $\frac{1}{2} \mathrm{XXZ}$-model chain as a function of the anisotropy parameter $\Delta$. We compare the exact results in the antiferromegnetic $(\Delta>-1)$ and ferromagnetic $(\Delta \leqslant-1)$ regimes with the uncorrelated Néel approximation $\left(E_{g} / N=-\frac{1}{4} \Lambda\right)$ and the present CCM results in the SUB2-2, SUB2, PSUB1, and LSUB-4 approximations described in the text. The terminating point at $\Delta=\Delta_{c} \approx 0.37275$ for the SUB2 scheme is clearly shown. We note that although the exact results show a phase change at $\Delta=1$, the energy is continuous and smooth at this point 
Thus, for large values of $m$ the leading asymptotic term in $b_{2 m-1}$ is given from Eqs. (34) $-(36)$ as:

$$
b_{2 m-1} \underset{m \rightarrow \infty}{\longrightarrow} \begin{cases}O\left(\xi^{-2 m}\right) ; & \Delta>\Delta_{c} \\ \gamma m^{-2} ; & \Delta=\Delta_{c},\end{cases}
$$

where $\xi$ is a function of $\Delta$ only, and $\gamma$ is a constant. This changeover from exponential to algebraic long-range decay is strongly reminiscent of the comparable behaviour of the exact spin-spin correlation function $G_{n}$ above and at the actual transition point $\Delta=1$, as given in Eq. (16).

We turn next to the PSUB1 approximation specified by Eq. (27). In this case the coupled set of $\mathrm{CCM}$ equations for the single-plaquette configuration coefficients $\left\{g_{n}\right\}$ may be derived as:

$$
\begin{gathered}
-\frac{1}{2}+\Delta g_{2}+\frac{3}{2} g_{2}^{2}-g_{4}=0, \\
\Delta g_{2 m}-g_{2 m+2}+2 m g_{2} g_{2 m}-\Delta \sum_{n=1}^{m-1} g_{2 n} g_{2 m-2 n}+\left(1-\delta_{m 2}\right) \sum_{n=2}^{m-1} g_{2 n} g_{2 m-2 n+2}=0 ; \\
m \geqslant 2 .
\end{gathered}
$$

The corresponding PSUB1 estimate for the ground-state energy is again given by Eq. (31), with $b_{1} \equiv g_{2}$. We have not sought an exact solution to Eqs. $(38 \mathrm{a}, \mathrm{b})$. However, just as for the SUB2-n sequence of approximations, so too is the convergence with respect to $n$ of the ground-state energy very rapid for the PSUB1- $n$ sequence for all values $\Delta>-1$ in the antiferromagnetic regime, as exemplified in Table 1. The (converged) numerical results are also displayed in Fig. 1. We see clearly that the PSUB1 estimate for the ground-state energy is superior to the SUB2 estimate over essentially the whole range $\Delta>\Delta_{c}$ for which comparison can be made. On the other hand, for smaller values of $\Delta$ the PSUB1 approximation both becomes increasingly inaccurate and, unlike the SUB2 approximation, shows no sign of terminating, and hence gives no signal of the physical antiferromagnetic phase transition.

The corresponding results for the ground-state energy from the LSUB-4 approximation are also displayed in Fig. 1. The use of Eq. (28) in this case leads to the three coupled equations:

$$
\begin{array}{r}
2 \Delta b_{1}-1+3 b_{1}^{2}-2 b_{1} b_{3}-2 b_{3}^{2}-2 g_{4}=0, \\
2 \Delta b_{3}-\frac{1}{2} b_{1}^{2}+2 b_{1} b_{3}-\frac{1}{2} g_{4}=0, \\
\Delta b_{1}\left(b_{1}+2 b_{3}\right)-2 b_{1} b_{3}^{2}-\left(\Delta+4 b_{1}+b_{3}\right) g_{4}=0,
\end{array}
$$

Table 1. The ground-state energy per spin, $E_{g} / N$, for the infinite $(N \rightarrow \infty)$ spin- $\frac{1}{2}$ XXZ-model chain in $1-\mathrm{d}$, for various values of the anisotropy parameter $\triangle$. CCM results for various PSUB1-n approximations are given and compared with corresponding exact values

\begin{tabular}{rllllll}
\hline$\Delta$ & \multicolumn{1}{l}{$E_{g} / N$} \\
\cline { 2 - 7 } & PSUB1-2 & PSUB1-4 & PSUB1-6 & PSUB1-8 & PSUB1-10 & Exact \\
\hline 5.0 & -1.29858 & -1.29944 & -1.29947 & -1.29947 & -1.29947 & -1.29950 \\
2.0 & -0.60762 & -0.61436 & -0.61509 & -0.61517 & -0.61517 & -0.61722 \\
1.0 & -0.41667 & -0.42966 & -0.43099 & -0.43111 & -0.43111 & -0.44315 \\
0.0 & -0.28867 & -0.28867 & -0.28867 & -0.28867 & -0.28867 & -0.31831 \\
-1.0 & -0.25000 & -0.16491 & -0.17599 & -0.17525 & -0.17529 & -0.25000 \\
\hline
\end{tabular}


Table 2. The ground-state energy per spin, $E_{g} / N$, for the infinite $(N \rightarrow \infty)$ spin- $-\frac{1}{2}$ XXZ-model chain in $1-\mathrm{d}$, for various values of the anisotropy parameter $\Delta$. CCM results in the LSUB-4 and SUB2 $+g_{4}$ approximation schemes are compared with exact results

\begin{tabular}{rlll}
\hline \multirow{2}{*}{$\Delta$} & \multicolumn{3}{l}{$E_{g} / N$} \\
\cline { 2 - 4 } & LSUB-4 & SUB2 $+g_{4}$ & Exact \\
\hline 5.0 & -1.29947 & -1.29947 & -1.29950 \\
2.0 & -0.61552 & -0.61553 & -0.61722 \\
1.0 & -0.43627 & -0.43657 & -0.44315 \\
0.5 & -0.36921 & -0.37270 & -0.37500 \\
0.0 & -0.31934 & - & -0.31831 \\
-1.0 & -0.32110 & - & -0.25000 \\
-2.0 & -0.50482 & - & -0.50000 \\
\hline
\end{tabular}

for the coefficients of the only three configurations retained in this approximation. In view of the extremely rapid convergence of both the previous SUB2 and PSUB1 schemes it might be expected that the LSUB-4 approximation should contain the most important contributions from each, despite its relative simplicity. It is clear from both Fig. 1 and Table 2 that this is borne out. More surprising is the fact that the LSUB-4 scheme even appears to give reasonable results in the ferromagnetic regime.

Finally, we note that in order to obtain a phase transition it is essential to include at least some of the important long-range correlations. It seems probable that a necessary condition for this is that terms of arbitrarily long range in the cluster correlation operator should be retained. Apparently the SUB2 approximation is at least qualitatively successful in this regard. Nevertheless, as we have seen from the LSUB-4 results, and as was also pointed out by Roger and Hetherington [39] for the Heisenberg model, the inclusion of other short-range correlations can improve the quantitative estimate of the ground-state energy considerably. We might therefore anticipate that an additional approximation scheme which incorporates both the long-range correlations of the SUB2 scheme and the one additional four-spin plaquette configuration contained in the LSUB4 scheme, could continue to exhibit a terminating point as a signal of the actual transition at $\Delta=1$, and also yield improved accuracy. Numerical calculations show that such is indeed the case for this so-called SUB2 $+g_{4}$ scheme, in which the terminating point now occurs at a value $\Delta_{c}^{\prime} \approx 0.4355$. For $\Delta>\Delta_{c}^{\prime}$, the corresponding ground-state energy estimates are even closer to the exact values than the LSUB-4 results, as can also be seen from Table 2 .

\section{NCCM parametrization of the ground bra state}

In order to calculate quantities other than the energy, we cannot make direct use of the Schrödinger equation in order to avoid the evaluation of an expectation value, and hence we need also to parametrize the bra state. Clearly the most straightforward way is simply to keep the bra and ket states as manifest Hermitian adjoints of each other. This method was employed independently by 
Čižek [9] and Fink [72], and it leads for an arbitrary operator $A$ to its expectation value $\bar{A}$ in the form:

$$
\begin{aligned}
\bar{A} & =\left\langle\Phi\left|e^{S \dagger} A e^{S}\right| \Phi\right\rangle\left\langle\left\langle\Phi\left|e^{S \dagger} e^{S}\right| \Phi\right\rangle,\right. \\
& =\left\langle\Phi\left|\left(e^{S \dagger} A e^{S}\right)_{\mathscr{L}}\right| \Phi\right\rangle .
\end{aligned}
$$

Whereas Eq. (40a) may be reduced to the sum in Eq. (40b) of linked $(\mathscr{L})$ diagrams, there is no such automatic termination upon expansion in powers of $S$ as occurs in Eq. (30). What is even worse is that if the infinite sum implied by Eq. (40b) is truncated, the resulting approximant for $\bar{A}$ is in conflict with the important Hellmann-Feynman theorem [73]. As Thouless [74] has shown, this implies that $\bar{A}$ should be calculated from the same set of diagrams as for the energy, but in which one of the interaction potential lines is replaced by the operator $A$ in all possible ways. Kümmel [75] has described a more elaborate method to calculate $\bar{A}$ within the CCM, but it is also in conflict with the above theorem.

The first formulation within the CCM of a method to evaluate $\bar{A}$ which is consistent with the Hellmann-Feynman theorem was given by Monkhorst [76]. He used techniques of linear and higher-order response theory, but never introduced an explicit average-value functional. His scheme was later supplemented by the incorporation of the basis set effects [77] which are vital for the practical analytical evaluation of energy derivatives in quantum chemistry. Indeed, this latter scheme has by now very successfully been used [78] to predict vibrational spectra and to locate transition states for decomposition reactions.

Finally in this context, Arponen [14] introduced two different explicit CCM parametrizations of the bra state. Neither is manifestly the Hermitian conjugate of the corresponding ket state for reasons outlined above. The main advantages that now accrue to such non-manifestly-Hermitian descriptions are that one can now give an explicit functional for an arbitrary expectation value $\bar{A}$ which is derivable from a variational principle, and which is compatible with the Hellmann-Feynman theorem at all levels. The first of these two methods corresponds to a generalization of the method of Monkhorst [76], and it has been named the normal coupled cluster method (NCCM).

The bra ground-state wavefunction $\langle\widetilde{\Psi}|$ corresponding to $|\Psi\rangle$, where $\langle\tilde{\Psi}| H=E_{g}\langle\tilde{\Psi}|$, is parametrized in the NCCM as:

$$
\langle\tilde{\Psi}|=\langle\Phi| \tilde{S} e^{-S},
$$

where $\tilde{S}$ is a new correlation operator which at this stage is formally independent of $S$, although in principle it may exactly be expressed as:

$$
\langle\Phi| \tilde{S}=\frac{\langle\Phi| e^{S \dagger} e^{S}}{\left\langle\Phi\left|e^{S \dagger} e^{S}\right| \Phi\right\rangle} .
$$

It is decomposed entirely in terms of destruction operators with respect to $|\Phi\rangle$, in the same way that the ket-state operator $S$ was decomposed in terms of creation operators. Thus, for the 1-d spin $-\frac{1}{2}$ chain considered here, we write by analogy with Eqs. (24) and (25):

$$
\tilde{S}=1+\sum_{\ell=1}^{L} \tilde{S}_{2 \ell},
$$




$$
\tilde{S}_{n+1}=\sum_{i=1}^{N} \sum_{\left\{r_{1}, r_{2}, \ldots, r_{n}\right\}} \tilde{s}_{r_{1} r_{2} \ldots r_{n}}^{(n+1)} \sigma_{i}^{-} \sigma_{i+r_{1}}^{-} \sigma_{i+r_{2}}^{-} \cdots \sigma_{i+r_{n}}^{-},
$$

where $N=2 L$, and where the $n$-fold sum over the ordered set of indices $\left\{r_{1}, r_{2}, \ldots, r_{n}\right\}$ in Eq. (44) has the same meaning as in Eq. (25). We note that the particular value (unity) of the constant term in Eq. (43) implies the manifest normalization, $\langle\tilde{\Psi} \mid \Psi\rangle=\langle\Phi \mid \Phi\rangle=1(\doteq\langle\Phi \mid \Psi\rangle)$. We note finally that although the NCCM expectation value $\bar{A}=\left\langle\Phi\left|\widetilde{S} e^{-S} A e^{S}\right| \Phi\right\rangle$ is composed wholly of linked terms, the operator $\tilde{S}$ itself does generally contain unlinked pieces, unlike the original wholly linked operator $S$. (In fact, it was to cure this remaining unlinkedness that Arponen also introduced the alternative extended coupled cluster method (ECCM) parametrization of the bra state.)

It is clear that although the exact operators $S$ and $\tilde{S}$ do preserve the Hermitian-conjugacy between corresponding ket and bra states, subsequent approximation schemes generally will not. In practical implementations of the NCCM, the operator $\tilde{S}$ is truncated by retaining exactly the same set of destruction cluster configurations as $S$ keeps their creation counterparts. The equations which determine the coefficients of the set of configurations retained in $S$ and $\tilde{S}$ are then derived by the requirement that the NCCM energy expectation value $\bar{H}=\left\langle\Phi\left|\tilde{S} e^{-S} H e^{S}\right| \Phi\right\rangle$ should be stationary with respect to every such coefficient [14].

A typical example is the SUB2 approximation in which the analogue of Eq. (26) for the infinite chain is $\widetilde{S} \rightarrow \tilde{S}_{\mathrm{SUB} 2}$ where:

$$
\tilde{S}_{\mathrm{SUB} 2} \equiv 1+\tilde{S}_{2}=1+\sum_{i=1}^{\infty} \sum_{m=1}^{\infty} \tilde{b}_{2 m-1} \sigma_{i}^{-} \sigma_{\bar{i}+2 m-1}^{-},
$$

and $\bar{H}$ is now a functional of both sets of amplitudes $\left\{b_{r}\right\}$ and $\left\{\tilde{b}_{r}\right\}$. The stationarity of $\bar{H}$ with respect to $\tilde{b}_{2 m-1}$ gives the set of formal equations:

$$
\left\langle\Phi\left|\sum_{i} \sigma_{i}^{-} \sigma_{i+2 m-1}^{-} e^{-s} H e^{S}\right| \Phi\right\rangle=0 ; \quad m \geqslant 1 .
$$

These equations, when evaluated with $S \rightarrow S_{\mathrm{SUB} 2}$, lead precisely to our earlier set of CCM SUB2 Eqs. (32a,b). Similarly, the stationarity of $\vec{H}$ with respect to $b_{2 m-1}$ yields the formal equations:

$$
\left\langle\Phi\left|\tilde{S} e^{-S}\left[H, \sum_{i} \sigma_{i}^{+} \sigma_{i+2 m-1}^{+}\right] e^{s}\right| \Phi\right\rangle=0 ; \quad m \geqslant 1,
$$

which have the form of a coupled set of linear equations for the coefficients $\left\{\tilde{b}_{2 m-1}\right\}$ once the ket-state coefficients $\left\{b_{2 m-1}\right\}$ are used as known input from the solution to Eq. (46), and hence to Eqs. (32a,b).

We remind the reader of two important points. Firstly, we note that the ground-state energy calculated by evaluating $\bar{H}=\left\langle\Phi\left|\tilde{S} e^{-S} H e^{S}\right| \Phi\right\rangle$ at the stationary point in this NCCM procedure is identical to our earlier relation $E_{g}=\left\langle\Phi\left|e^{-S} H e^{S}\right| \Phi\right\rangle$ from Eq. (30) at any level of approximation, due to the stationarity conditions satisfied by the bra-state amplitudes. Secondly, we note that despite the fact that the ground-state energy has now been evaluated from a variational principle, the corresponding estimate from any particular truncation scheme does not in general yield an upper bound to the true energy, since the formalism does not manifestly preserve the Hermitian conjugacy between the bra and ket states. 
In the case of the SUB2 approximation, explicit evaluation of Eq. (47) yields the coupled equations:

$$
\begin{array}{rl}
\delta_{m 1}+2\left(\delta_{m 1}-2\right)\left(\Delta+2 b_{1}\right) \tilde{b}_{2 m-1}-8 \delta_{m 1} \sum_{n=1}^{\infty} \tilde{b}_{2 n-1} b_{2 n-1} & \\
+2 \sum_{n=1}^{\infty} \tilde{b}_{2 n-1}\left(b_{|2 m-2 n-1|}+b_{|2 m-2 n+1|}+b_{2 m+2 n-1}+b_{2 m+2 n-3}\right)=0 & m \geqslant 1,
\end{array}
$$

as the bra-state counterparts of Eqs. (32a,b). These equations may also be evaluated in any SUB2- $n$ subapproximation. Thus, for example, Eqs. (32a) and (48) yield the SUB2-2 solutions:

$$
b_{1}=\frac{1}{3}\left[\left(\Delta^{2}+3\right)^{1 / 2}-\Delta\right], \quad \tilde{b_{1}}=\frac{1}{2}\left(\Delta^{2}+3\right)^{-1 / 2} ; \quad \text { SUB2-2. }
$$

Furthermore, the full set of coupled Eqs. (48) may again be solved by Fourier transform techniques to give, as the counterpart of Eq. (34):

$$
\tilde{b}_{2 m-1}=\frac{D}{4 K} \frac{1}{2 \pi} \int_{-\pi}^{\pi} d x \frac{\cos \left[\left(m-\frac{1}{2}\right) x\right] \cos \left(\frac{1}{2} x\right)}{f\left(x ; k^{2}\right)},
$$

where the function $f\left(x ; k^{2}\right)$ is as defined previously in Eq. (35), and where:

$$
D^{-1}=\frac{1}{2 \pi} \int_{-\pi}^{\pi} d x f^{-1}\left(x ; k^{2}\right) f^{2}\left(x ; \frac{1}{2}\right)-\frac{1}{2} .
$$

In this case, the leading asymptotic term in $\tilde{b}_{2 m-1}$ for large values of $m$ is given from Eqs. (50) and (51) as:

$$
\tilde{b}_{2 m-1} \underset{m \rightarrow \infty}{\longrightarrow} \begin{cases}O\left(\lambda^{-2 m}\right) ; & \Delta>\Delta_{\mathfrak{c}} \\ \Gamma ; & \Delta=\Delta_{c},\end{cases}
$$

where $\lambda$ is a function of $\Delta$ only, and $\Gamma$ is a constant.

\section{NCCM treatment of properties; staggered magnetization and correlation function}

Given any approximate NCCM solution for the bra and ket ground-state wavefunctions, it is a straightforward matter to calculate the corresponding approximation for any ground-state property. One of the most interesting properties to study first, particularly in connection with the possible phase transition indicated by the terminating point at $\Delta=\Delta_{c}$ in the SUB2 approximation, for example, is the staggered magnetization. This quantity is defined (in the rotated Néel basis) in Eq. (15). Thus, by inserting the SUB2 parametrizations of Eqs. (26) and (45) into the NCCM expectation value functional for the staggered magnetization vector $M^{\alpha}(\alpha=x, y, z)$, we easily find:

$$
M^{\alpha}=-M \delta_{\alpha z},
$$

where:

$$
M=1-4 \sum_{m=1}^{\infty} \tilde{b_{2 m-1}} b_{2 m-1} ; \quad \text { SUB2. }
$$

By restricting the sum in Eq. (54) to the $m=1$ term only, and by making use 


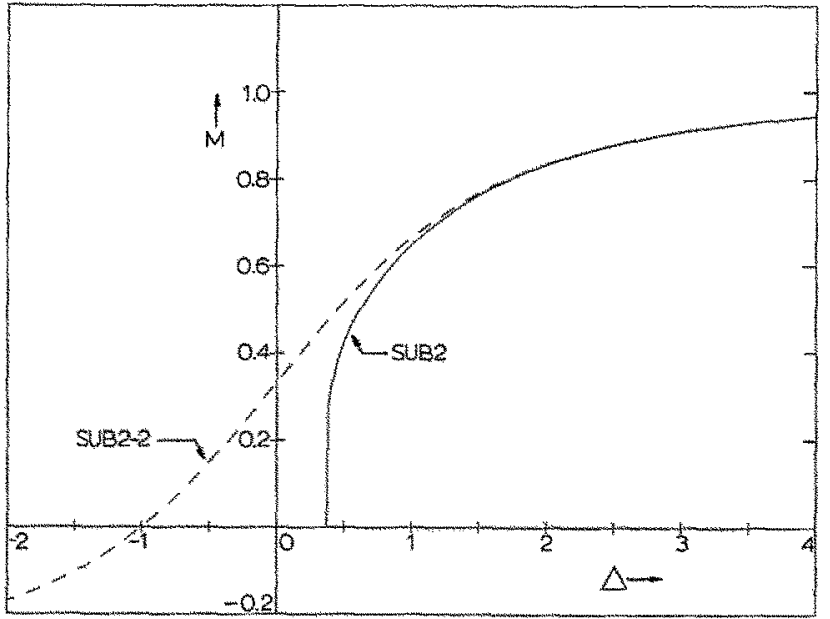

Fig, 2. The staggered magnetization, $M$, as a function of the anisotropy parameter $A$ for the infinite $\operatorname{spin}-\frac{1}{2} \times X Z$-model chain for the two NCCM approximations SUB2-2 and SUB2. We note that the SUB2 scheme only has solution for $\Delta>\Delta_{c} \approx 0.37275$, the point at which $M \rightarrow 0$ in this approximation

of Eq. (49), we first obtain the SUB2-2 estimate:

$$
M_{\text {SuBz-2 }}=\frac{1}{3}\left[1+2 \Delta\left(A^{2}+3\right)^{-\frac{1}{2}}\right] \text {. }
$$

The full sum in Eq, (54) may also be evaluated from the explicit solutions of Eqs. (34) $-(36)$ and $(50)-(51)$. We thus find the full SUB2 result for the staggered magnetization:

$$
M_{\mathrm{SUB} 2}=\frac{\frac{1}{\pi} \int_{0}^{\pi} d x \sin ^{2}\left(\frac{1}{2} x\right) f^{-1}\left(x ; k^{2}\right)}{\frac{1}{\pi} \int_{0}^{\pi} d x\left[1+\sin ^{2}\left(\frac{1}{2} x\right)\right] f^{-1}\left(x ; k^{2}\right)-1}
$$

These two estimates from Eqs. (55) and (56) of the staggered magnetization $M$ are shown in Fig. 2. In particular, we observe that $M_{\mathrm{SuB} 2} \rightarrow 0$ as $A \rightarrow \Delta_{c}$, the terminating point of the SUB2 approximation, just as we expect of the exact phase transition at $A=1$. Both of the SUB2 and SUB2-2 approximants have the same asymptotic form, $M \rightarrow 1-\Delta^{-2}$ in the Ising-model limit, $\Delta \rightarrow \infty$.

Further support for the behaviour of the SUB2 approximation as $\Delta \rightarrow \Delta_{c}$ being a signal of the actual phase transition, is provided by the spin-spin correlation function $G_{n}$ defined in $\mathrm{Eq}$. (14a). For these purposes it is actually sufficient to consider only its $z z$-component, $G_{n}^{z z} \equiv\left\langle\sigma_{i}^{z} \sigma_{i+n}^{z}\right\rangle ; n \geqslant 0$. In the SUB2 NCCM approximation it is again not difficult to evaluate this correlation function explicitly. We find the result:

$$
G_{n}^{z z}=1+4 \bar{b}_{n} b_{n}-8\left(1-\delta_{n p_{0}}\right) \sum_{m=1}^{\infty} \bar{b}_{2 m-1} b_{2 m \ldots 1} ; \quad \text { SUB2. }
$$

Since the SUB 2 coefficients $\left\{b_{n}\right\}$ and $\left\{\bar{b}_{n}\right\}$ are nonzero only for odd values of $n$ in the $s_{T}^{z}=0$ subspace to which we have confined ourselves, it is clear that the SUB2 estimate for $G_{n}^{z z}$ is independent of $n$ for $n$ even and nonzero. On the other hand, for $n$ odd we observe the same longrange $(n \rightarrow \infty)$ SUB2 value, $G_{n}^{z z} \rightarrow \mu=2 M-1$ as the constant value between different sites on the same sublattice, in this approximation. Furthermore, for $n$ odd we observe from Eqs. 
(37), (52) and (57) that the long-range decay in the $z z$-correlations between spins on different sublattices exhibits precisely the same changeover from exponential (for $\Delta>\Delta_{c}$ ) to algebraic (at $\Delta=\Delta_{c}$ ) behaviour as characterizes the exact transition from the ordered phase $(\Delta>1)$ to the critical phase $(|\Delta|<1)$, from Eq. (16).

\section{NCCM parametrization of excited ket states; excitation energies}

Finally, we may also examine the elementary excitations of the system as a function of $\Delta$. Of special interest in this context is whether or not the excitation spectrum exhibits a gap. We employ the usual CCM technique of Emrich [79] for constructing the excited-state ket wavefunction $\left|\Psi_{e}\right\rangle$ in terms of a linear excitation operator $X$ which acts on the corresponding ground-state wavefunction $|\Psi\rangle$ :

$$
\left|\Psi_{e}\right\rangle=X|\Psi\rangle=X e^{S}|\Phi\rangle \text {. }
$$

The excitation operator $X$ is chosen, like the ground-state correlation operator $S$, to be constructed purely from linear combinations of products of single-spin creation operators:

$$
\begin{aligned}
X & =\sum_{n=1}^{N} X_{n}, \\
X_{n} & =\sum_{\left\{r_{1}, r_{2}, \ldots, r_{n}\right\}} x_{r_{1} r_{2} \ldots r_{n}}^{(n)} \sigma_{r_{1}}^{+} \sigma_{r_{2}}^{+} \cdots \sigma_{r_{n}}^{+},
\end{aligned}
$$

where the sum over the ordered set of indices $\left\{r_{1}, r_{2}, \ldots, r_{n}\right\}$ is as given in Eq. (25). The operators $X$ and $S$ thus commute. The simplest possible approximation for $X$ is the replacement:

$$
X \rightarrow X_{1}=\sum_{s} x_{s} \sigma_{s}^{+},
$$

where the sum on the index $s$ runs over all sites of a given sublattice. Furthermore, if the SUB2 approximation $\left(S \rightarrow S_{2}\right)$ for $|\Psi\rangle$ is also used in Eq. (58), the corresponding approximation for the excited states is henceforth denoted as the $\operatorname{SUB}(1,2)$ approximation. It is clear that the excitations so described will have $s_{T}^{z}= \pm 1$, since our SUB2 approximation for $|\Psi\rangle$ has $s_{T}^{z}=0$.

By combining the excited-state Schrödinger equation, $H\left|\Psi_{e}\right\rangle=E_{e}\left|\Psi_{e}\right\rangle$, with its ground-state counterpart, we easily derive the general CCM equation for the excitation energy, $\varepsilon_{e} \equiv E_{e}-E_{g}$ :

$$
e^{-S}[H, X] e^{S}|\Phi\rangle=\varepsilon_{e} X|\Phi\rangle .
$$

A coupled set of linear equations for the various configuration coefficients of the operator $X$ is now obtained by taking the inner product of Eq. (61) in turn with the respective wavefunctions corresponding to each configuration (with respect to the Néel state) retained in Eq. (59) by the specific approximation. In each of these equations the corresponding approximate CCM solution for the groundstate operator $S$ is used as known input. The resulting equations have the form of a set of linear eigenvalue equations. Equivalently, we observe that the approximate CCM excitation energies $\varepsilon_{e}$ are simply obtained by diagonalizing the operator $\left(e^{-s} H e^{S}-E_{g}\right)$, where $S$ is the approximated CCM ground-state correlation operator, within the subspace formed by states of the form of those 
retained in Eq. (59). In the present $\operatorname{SUB}(1,2)$ case, for example, the coefficients $\left\{x_{s}\right\}$ are simply the components of the corresponding eigenvectors belonging to each such eigenvalue $\varepsilon_{e}$ within the subspace of vectors $\left\{\sigma_{s}^{+}|\Phi\rangle\right\}$.

For our infinite $1-\mathrm{d}$ spin- $\frac{1}{2}$ chain, the resulting $\operatorname{SUB}(1,2)$ equations, which are thus easily derived by taking the inner product of Eq. (59) with the state $\sigma_{s}^{+}|\Phi\rangle$, may once again be solved by Fourier transform techniques. In this way the individual excited states may now be labelled by a wave vector $q$ such that $-\pi<q \leqslant \pi$, and the excitation energy spectrum $\varepsilon_{e}$ becomes $\varepsilon(q)$. The final result is given by:

$$
\varepsilon(q)=K\left(1-k^{2} \cos ^{2} q\right)^{\frac{1}{2}} ; \quad-\pi<q \leqslant \pi ; \quad \operatorname{SUB}(1,2)
$$

where the constants $k^{2}$ and $K$ are defined in Eq. (36). As we have described in Sect. $3, k^{2}<1$ for $\Delta>\Delta_{c}$, and the terminating value $\Delta=\Delta_{c}$ is characterized by the point at which $k^{2}=1$. Hence, the excitation spectrum of Eq. (62) has a gap for $\Delta>\Delta_{c}$, but when $\Delta=\Delta_{c}$ it has the form $\varepsilon_{c}(q)=K_{c} \sin (q)$, where $K_{c} \approx 1.3642$. It is clear that this behaviour closely reflects that of the exact excitation spectrum above and at the actual transition point $\Delta=1$. For example, Eq. (18) shows that the exact single-kink $\left(s_{T}^{z}=\frac{1}{2}\right)$ spectrum has the form $\varepsilon_{c}(q)=\frac{1}{2} \pi \sin (q)$ at $\Delta=1$. Furthermore, this same expression is also precisely the lower $(k=0)$ boundary, $E_{0}^{c}(q)=\varepsilon_{c}(q)$, of the physical two-kink $\left(s_{T}^{z}= \pm 1\right)$ continuum given by Eq. (22) in the case $\Delta=1$.

\section{Discussion and conclusions}

We believe that our analytic and numerical results for the infinite spin- $\frac{1}{2}$ XXZ-model chain in 1-d clearly indicate that the CCM techniques and approximation schemes that we have described have the potential to be powerful tools for use on a wider class of related spin-lattice problems. In particular, the important extension to the spin- $\frac{1}{2}$ square lattice in $2-\mathrm{d}$ is relatively straightforward. Nevertheless, in this case the possible approximation schemes are more complicated. Although we cannot expect that the most important 2-d "wrongspin" configurations will be the same for all values of $\Delta$, there are at least two features that we expect to be physically significant in guiding us to formulate appropriate truncation schemes, at least in the region where the quantum fluctuations have not wholly destroyed the long-range order of the Néel state.

Thus, in the first place we expect that configurations with a small number of "wrong bonds" will be more important than those with a higher number, since in a classical picture the breaking of each Néel-state bond in the antiferromagnetic regime costs energy. The second concept is based on an extension to 2-d of the seemingly important aspects in 1-d of the number of kinks present in a configuration and the size of the locale. We are thus led to define the length of the "domain boundary" of a given configuration as the number of lattice bonds crossed by a shortest-path circuit enclosing all of the "wrong" spins of that configuration. We then expect that configurations with the smallest value of this domain-boundary length may be of predominant importance. More generally, those configurations with the least values of both of the above parameters are almost sure to be the most significant.

We have performed some preliminary calculations for the 2-d square lattice which seems to bear out the relevance of the above two points, at least for calculations of the ground-state energy. Furthermore, calculations in the SUB2 
approximation based on a Néel model state again give a terminating point. The corresponding critical value, $\Delta_{c} \approx 0.7985$, is thus closer to the classical value of 1 than for the 1-d case. However, quite unlike the 1-d case, the staggered magnetization for the square lattice does not vanish in the NCCM SUB2 approximation at $\Delta=\Delta_{c}$, but instead takes the value $M_{c} \approx 0.682$, at which point the ground-state energy has the value $E_{g} / N \approx-0.5836$. By contrast, we note that spin-wave theory $[67,68,80]$, for example, breaks down in all dimensions whenever $\Delta<1$. For the $1-\mathrm{d}$ chain spin-wave theory gives a divergent result at $\Delta=1$ for the staggered magnetization, whereas the comparable result for the 2-d lattice is $M_{c} \approx 0.606$. Our own SUB2 results for the Heisenberg square lattice are $E_{g} / N \approx-0.6508$ and $M \approx 0.827$.

For better comparison purposes it is unfortunate that very few exact results are known for the spin- $\frac{1}{2}$ lattice for dimensionality greater than one. For example, to the best of our knowledge, no rigorous proof has ever been given concerning the presence of long-range order for the 2-d Heisenberg model on a square lattice, although it is known that this model is disordered at any nonzero temperature [81]. However, on the basis of numerical work involving both exact diagonalization on small lattices and Monte Carlo calculations of various kinds for bigger lattices, it is widely believed that the spin- $-\frac{1}{2}$ Heisenberg model on a square lattice shows a nonzero staggered magnetization, unlike its $1-\mathrm{d}$ chain counterpart.

For example, Green's function Monte Carlo calculations have been performed for the Heisenberg model by both Carlson [82] and Trivedi and Ceperley [83], on lattices of size up to $32 \times 32$. These authors agree that the extrapolated ground-state energy for infinite lattice size is $E_{g} / N=-0.6692 \pm 0.0002$, whereas for the staggered magnetization (in our units) Carlson gives $M=0.68 \pm 0.02$ and Trivedi and Ceperley give $M=0.62 \pm 0.04$. In conclusion, it is clear that our own CCM results suggest the presence of a phase transition for the 2-d XXZ-model on a square lattice also, but with some features which are quite different from the 1-d chain. Although more work is clearly needed, we are encouraged by these early results.

It will also be of interest to extend our $1-d$ spin- $\frac{1}{2}$ chain results to the corresponding cases with spins $s>\frac{1}{2}$, particularly in view of the possibility to shed some light on the appearance of the so-called Haldane phase [59-61] for the spin-1 chain. We are also interested in studying the sensitivity of the present results and of particular approximation schemes to the choice of model state. Although the Néel state is an obvious candidate in this respect, it is by no means the only such choice.

Finally, we hope that the new PSUB $n$ and LSUB- $n$ schemes that we have devised may also find wider practical use. It is possible that not only might they be useful for application to such other discrete lattice problems of interest to chemists as the cyclic polyenes, but also to more general molecular systems.

Acknowledgements. One of us (R.F.B.) gratefully acknowledges support for this work in the form of a research grant from the Science and Engineering Research Council (SERC) of Great Britain.

\section{References}

1. Bednorz JG, Müller KA (1986) Z Phys B 64:189

2. Anderson PW (1987) Science 235:1196 
3. Hubbard J (1964) Proc Roy Soc London A276:238; idem (1965) ibid A285:542

4. Baskaran G, Zou Z, Anderson PW (1987) Solid State Commun 63:973

5. Kalmeyer V, Laughlin RB (1987) Phys Rev Lett 59:2095

6. Affleck I, Marston JB (1988) Phys Rev B 37:3774

7. Bishop RF, Kümmel HG (1987) Phys Today 40(3):52

8. Coester F (1958) Nucl Phys 7:421; Coester F, Kümmel H (1960) Nucl Phys 17:477

9. Čižek J (1966) J Chem Phys 45:4256; idem (1969) Advan Chem Phys 14:35

10. Kümmel H, Lührmann KH, Zabolitzky JG (1978) Phys Rep 36C:1

11. Bishop RF, Lührmann KH (1978) Phys Rev B 17:3757; idem (1982) ibid 26:5523

12. Bartlett RJ (1981) Annu Rev Phys Chem 32:359; idem (1989) J Phys Chem 93:1697

13. Kvasnička V, Laurinc V, Biskupič S (1982) Phys Rep 90C:159

14. Arponen J (1983) Ann Phys (NY) 151:311

15. Arponen J, Bishop RF, Pajanne E (1987) Phys Rev A 36:2519, 2539; Arponen J, Bishop RF, Pajanne E, Robinson NI (1988) ibid 37:1065

16. Clark JW, Feenberg E (1959) Phys Rev 113:388

17. Jackson HW, Feenberg E (1961) Ann Phys (NY) 15:266

18. Clark JW, Westhaus P (1966) Phys Rev 141:833

19. Fantoni S, Rosati S (1974) Lett Nuovo Cim 10:545; idem (1975) Nuovo Cim 25A:593

20. Krotscheck E, Ristig ML (1974) Phys Lett 48A:17; idem (1975) Nucl Phys A242:389

21. Clark JW (1979) Prog Part Nucl Phys 2:89

22. Krotscheck E, Clark JW (1980) Nucl Phys A333:77

23. Clark JW (1981) in: Guardiola R, Ros J (eds) The many-body problem, Jastrow correlations versus Brueckner theory. Springer, Berlin, p 184

24. Krotscheck E, Smith RA, Clark JW, Panoff RM (1981) Phys Rev B 24:6383; Flynn MF, Clark JW, Krotscheck E, Smith RA, Panoff RM (1985) ibid 32:2945

25. Krotscheck E, Clark JW, Jackson AD (1983) Phys Rev B 28:5088

26. Clark JW, Krotscheck E, Schwesinger B (1984) Phys Lett 143B:287; idem (1985) Anales Fisica A81:116

27. Bijl A (1940) Physica (Utrecht) 7:860; Jastrow R (1955) Phys Rev 98:1479

28. Jackson AD, Lande A, Smith RA (1982) Phys Rep 86:55; idem (1985) Phys Rev Lett 54:1469

29. Jackson AD, Lande A, Guitink RW, Smith RA (1985) Phys Rev B 31:403

30. Krotscheck E, Smith RA, Jackson AD (1986) Phys Rev A 33:3535

31. Jackson AD, Smith RA (1987) Phys Rev A 36:2517; Smith RA, Jackson AD (1988) Nucl Phys A476:448

32. Fantoni S, Wang X, Tosatti E, Lu Yu (1988) Physica C 153-155:1255; Wang XQ, Fantoni S, Tosatti E, Lu Yu (1990) Correlated spin-density-wave theory. In: Aguilera-Navarro VC (ed) Condensed matter theories, vol 5. Plenum, New York

33. Ristig ML (1990) Z Phys B 79:351

34. Dabringhaus A, Ristig ML (1989) The U(1) lattice gauge model: a correlated many-body system. In: Keller J (ed) Condensed ; : ter theories, vol 4. Plenum, New York; idem (1991) The U(1) lattice gauge vacuum. In: Fantc S, Rosati S (eds) Condensed matter theories, vol 6. Plenum, New York

35. Paldus J, Čižek J, Takahashi M (1984) Phys Rev A 30:2193; Paldus J, Takahashi M, Cho RWH (1984) Phys Rev B 30:4267; Takahashi M, Paldus J (1985) ibid 31:5121

36. Piecuch P, Zarrabian S, Paldus J, Čižek J (1990) Phys Rev B 42:3351

37. Pauncz R, de Heer J, Löwdin P-O (1962) J Chem Phys 36:2247, 2257; de Heer J, Pauncz R (1960) J Mol Spectrosc 5:326; Pauncz R (1967) Alternant molecular orbital method. Saunders, Philadelphia

38. Parr RG (1963) The quantum theory of molecular electronic structure. Benjamin, New York

39. Roger M, Hetherington JH (1990) Phys Rev B 41:200

40. Lieb EH, Mattis DC (1966) Mathematical physics in one dimension. Academic, New York

41. Schuster HG (ed) (1975) One-dimensional conductors. (Lect Notes Phys, vol 34) Springer, Berlin

42. Keller HJ (ed) (1977) Chemistry and physics of one-dimensional metals. Plenum, New York

43. Barišić S, Bjeliš A, Cooper JR, Leontić B (eds) (1979) Quasi-one-dimensional conductors I and II. (Lect Notes Phys, vols 95 and 96) Springer, Berlin 
44. Devreese JT, Evrard RP, Van Doren VE (eds) (1979) Highly conducting one-dimensional solids. Plenum, New York

45. Bernasconi J, Schneider T (eds) (1980) Physics in one dimension. Springer, Berlin

46. Alcacer J (ed) (1980) The physics and chemistry of low dimensional solids. Reidel, Dordrecht, The Netherlands

47. André JM, Bredas JL, Balhalle J, Ladik J, Leroy G, Moser C (eds) (1980) Recent advances in the quantum theory of polymers (Lect Notes Phys, vol 113) Springer, Berlin

48. Jerome D, Schultz HJ (1982) Adv Phys 31:299

49. Monceau P (ed) (1985) Electronic properties of inorganic quasi-one-dimensional compounds. Reidel, Dordrecht, The Netherlands

50. Bethe HA (1931) Z Phys 71:205

51. Gaudin M (1983) La fonction d'onde de Bethe. Masson, Paris

52. Faddeev LD (1980) Soviet Sci Reviews, Contemp Math Phys C1:107

53. Kulish PP, Sklyanin EK (1979) Phys Lett 70A:461; Faddeev LD, Takhtajan LA (1979) Usp Mat Nauk 34:13

54. Orbach R (1958) Phys Rev 112:309

55. Yang CN, Yang CP (1966) Phys Rev 150:321, 327; idem (1966) ibid 151:258

56. Gochev IG (1971) Zh Eksp Teor Fiz 61:1674 [transl. as: Sov Phys-JETP 34:892]

57. Lieb EH, Wu FY (1968) Phys Rev Lett 20:1445

58. Hashimoto K, Čižek J, Paldus J (1988) Int J Quantum Chem 34:407

59. Haldane FDM (1983) Phys Lett 93A:464; idem (1983) Phys Rev Lett 50:1153

60. Affleck I (1985) Nucl Phys B257:397

61. Affleck I, Haldane FDM (1987) Phys Rev B 36:5291

62. Endoh Y, Shirane G, Birgeneau RJ, Richards PM, Holt SL (1974) Phys Rev Lett 32:170; Hellmann IU, Shirane G, Endoh Y, Birgeneau RJ, Holt SL (1978) Phys Rev B 18:3530

63. Bishop RF, Arponen JS (1990) Int J Quantum Chem: Quantum Chem Symp 24:197

64. Bishop RF, Arponen J, Pajanne E (1989) Dynamic variational principles and extended coupled cluster techniques. In: Mukherjee D (ed) Aspects of many-body effects in molecules and extended systems (Lect Notes Chem, vol 50) Springer, Berlin, p 79

65. Lieb E, Schultz T, Mattis D (1961) Ann Phys (NY) 16:407; Lieb EH, Mattis DC (1962) J Math Phys 3:749

66. Hulthén L (1938) Arkiv Mat Astron Fysik 26A: No. 11

67. Anderson PW (1952) Phys Rev 86:694

68. Kubo R (1952) Phys Rev 87:568

69. des Cloiseaux J, Pearson JJ (1962) Phys Rev 128:2131

70. des Cloiseaux J, Gaudin M (1966) J Math Phys 7:1384

71. Faddeev LD, Takhtajan LA (1981) Phys Lett 85A:375

72. Fink M (1974) Nucl Phys A221:163

73. Hellmann H (1934) Proc Acad Sci USSR 3:444; Feynman RP (1939) Phys Rev 56:340

74. Thouless DJ (1961) The quantum mechanics of many-body systems. Academic, New York

75. Kümmel HG (1983) Int J Quantum Chem 24:79

76. Monkhorst HJ (1977) Int J Quantum Chem: Quantum Chem Symp 11:421

77. Bartlett RJ (1986) in: Jørgensen P, Simon J (eds) Geometrical derivatives of energy surfaces and molecular properties. Reidel, Dordrecht, p 35; Salter EA, Trucks GW, Bartlett RJ (1989) J Chem Phys 90:1752

78. Scheiner AC, Scuseria GE, Rice JE, Lee TJ, Schaefer HF (1987) J Chem Phys 87:5361

79. Emrich K (1981) Nucl Phys A351:379, 397

80. Oguchi T (1960) Phys Rev 117:117

81. Mermin ND, Wagner H (1966) Phys Rev Lett 1133

82. Carlson J (1989) Phys Rev B 40:846

83. Trivedi N, Ceperley DM (1990) Phys Rev B 41:4552 